\title{
Análise da Relação entre a Precipitação Média do Reservatório Orós, Brasil - Ceará, e os Índices PDO e AMO Através da Análise de Changepoints e Transformada de Ondeletas
}

\author{
Renan Vieira Rocha ${ }^{1}$ (D), Francisco de Assis de Souza Filho ${ }^{1}$, Samiria Maria Oliveira da Silva ${ }^{1}$ \\ ${ }^{I}$ Departamento de Engenharia Hidráulica e Ambiental, Universidade Federal do Ceará, \\ Fortaleza, CE, Brasil.
}

Recebido em: 19 de Janeiro de 2018 - Aceito em: 30 de Agosto de 2018

\begin{abstract}
Resumo
Os riscos de extremos hidrológicos para um local ou região estão associados aos modos de variação do clima, em suas diversas escalas temporais. A compreensão da variabilidade de baixa frequência ganha uma elevada importância em regiões onde eventos de seca são frequentes, por estar associada a longos períodos de secas consecutivas. O presente artigo analisou a relação entre a precipitação média da bacia a montante da estação fluviométrica de Iguatu, com os índices PDO e AMO (Pacific Decadal Oscillation e Atlantic Multidecadal Oscillation) através das metodologias de Análise de changepoint, Transformada de Ondeletas (TO), Transformada de Ondeletas Cruzadas (XTC) e Análise da Coerência das Ondeletas (WTC). Essa estação mensura as vazões afluente ao reservatório de Orós, um dos principais do Estado do Ceará (Brasil). A precipitação média da bacia foi obtida a partir de dados de pluviômetros. Os resultados permitiram estabelecer uma relação entre a precipitação da região e os índices PDO e AMO, indicando que um modelo baseado nos índices pode ter alguma capacidade preditiva do comportamento da precipitação local. Nota-se também que períodos com fases simultaneamente positivas (negativas) da PDO e da AMO resultam em um comportamento mais previsível das precipitações da região, com valores abaixo (acima) do normalmente esperado.
\end{abstract}

Palavras-chave: PDO, AMO, precipitação, Ceará.

\section{Analysis of the Relationship Between the Average Rainfall of Orós Reservoir, Brazil - Ceará, and the PDO and AMO Indexes Through Changepoint Analysis and Wavelet Transform}

\begin{abstract}
The risks of hydrological extremes for a location or region are associated with modes of climate variation, at their various time scales. The understanding of low frequency variability gains a high importance in regions where drought events are frequent, due to its association with long periods of consecutive droughts. This paper analyzed the relationship between the average rainfall of the Iguatu station, to the PDO (Pacific Decadal Oscillation) and AMO (Atlantic Multidecadal Oscillation) indexes, through the methodologies of Changepoint Analysis, Wavelet Transform (TO), Cross Wavelet Transform (XTC) and Wavelet Coherency Analysis (WTC). This station measures the inflow of the Orós reservoir, one of the most important in the state of Ceará (Brazil). The average rainfall of the basin was obtained from data of rain gauges. The results allowed to establish a relationship between the precipitation of the region and the PDO and AMO indexes, indicating that a model based on the indexes may have some predictive capacity for the local precipitation behavior. It is also noted that periods with simultaneously positive (negative) phases of the PDO and the AMO results in a more predictable behavior of the region's precipitation, with values below (above) the normally expected.
\end{abstract}

Keywords: PDO, AMO, precipitation, Ceará.

Autor de correspondência: Renan Vieira Rocha, renanvierocha@gmail.com. 


\section{Introdução}

Os riscos de extremos hidrológicos para um local ou região estão associados aos modos de variação do clima, em suas diversas escalas temporais (sazonal, interanual, multidecadal e centenária), que determinam, por exemplo, a duração e severidade de eventos de seca. A partir da compreensão desses modos é possível quantificar os riscos associados e definir mecanismos para gerenciá-los.

A compreensão da variabilidade de baixa frequência (multidecadal) ganha uma elevada importância em regiões onde eventos de seca são frequentes, por estar associada a longos períodos de secas consecutivas. A presença de padrões de variação de baixa e média frequência na pluviometria já foi identificada por alguns estudos em regiões com as características citadas e associada a fenômenos naturais como os ciclos solares e flutuações de anomalias da temperatura da superfície do mar (TSM) (Silveira et al. 2015). Entre as flutuações de baixa frequência se destacam a Oscilação Decadal do Pacífico (Pacific Decadal Oscillation - PDO) e a Oscilação Multidecadal do Attântico (AMO - Atlantic Multi-decadal Oscillation)

A PDO é o principal componente da variabilidade da temperatura na superfície do mar (TSM) do Oceano Pacífico (Mantua et al. 1997), essa variabilidade é mensurada pelo índice homônimo, que mede a anomalia da intensidade da PDO, do pacífico norte, em relação aos valores médios históricos através do número de desvios padrões (Castro; Souza Filho e Silveira, 2013). De acordo com Silva e Galvíncio (2011), a PDO se comporta de forma oposta entre a TSM ao longo da costa oeste da América do Norte em relação ao restante do Pacífico Norte.

A AMO é baseada na anomalia média de TSM do Atlântico Norte, cujo índice homônimo é usualmente calculado removendo a tendência da TSM de forma a retirar os sinais decorrentes as mudanças climáticas, podendo também ser suavizada por uma média móvel de 10 anos (Einfield; Mestas-Nuñez e Trimble, 2001). A AMO é o principal mecanismo de variação climática de baixa frequência do Oceano Atlântico.

A identificação e quantificação dos impactos de eventos globais de larga escala em eventos em escala reduzida se revela uma tarefa difícil devido à complexidade dos fenômenos naturais, exigindo nas análises de séries temporais de índices climáticos e variáveis hidrológicas a utilização de ferramentas adequadas.

A Transformada de Ondeletas (TO) é uma ferramenta extensamente empregada para a análise de séries temporais por proporcionar a decomposição de uma série não estacionaria em um espaço de tempo e frequência, permitindo a determinação dos modos de variabilidade dominantes e como eles variam ao longo do tempo (Torrence e Compo, 1998).

A Transformada de Ondeletas Cruzada (XTC) é uma extensão bivariada da $\mathrm{TO}$, utilizada para compreender a relação entre duas séries temporais, em conjunto com a Análise de Coerência de Ondeletas (WTC). Trabalhos recentes empregaram essas ferramentas (TO, XTC e WTC), isoladamente ou em conjunto, buscando a identificação de mecanismos forçantes e um melhor entendimento das teleconexões entre eventos hidrológicos e padrões climáticos de larga escala, especificamente PDO e/ou AMO (Chang et al. 2017; Kuss e Gurdak, 2014; Novello et al. 2012; Tang et al. 2014; Vásquez et al. 2018; Wang et al. 2017).

Além da decomposição da série, outra abordagem consiste em estimar os pontos, ou ponto, no qual as propriedades estatísticas de uma série de observações se modificam (changepoints). A metodologia do changepoint já foi empregada em diversos estudos de séries temporais, especificamente para séries de precipitação, a abordagem de Killick e Eckley (2014), que busca a identificação dos pontos através da minimização de uma função de custo, já foi utilizada para verificação de mudanças em tendências e na disponibilidade de água (Andam-Akorful et al. 2017; Deng et al. 2018).

O presente trabalho escolheu como área de estudo a bacia hidrográfica a montante da estação fluviométrica de Iguatu, localizada no estado do Ceará, Brasil. Esse estado enfrentou, em 2017, uma seca meteorológica histórica, iniciada em 2012 e que acarretou na redução significativa dos estoques dos reservatórios, de $68 \%$, em janeiro de $2012\left(12.785 \mathrm{hm}^{3}\right)$ para 9,1\% em outubro de 2017 $\left(1.690 \mathrm{hm}^{3}\right)$ (FUNCEME, 2017).

Destaca-se na região a utilização de modelos chuvavazão como uma ferramenta para simulação das vazões afluentes aos principais reservatórios, buscando a construção dos possíveis cenários futuros. Esses modelos têm como principal variável de entrada a precipitação média da bacia hidrográfica cujas vazões se deseja simular, juntamente com o seu histórico de vazões, para calibração e validação do modelo. A estimação precisa dos valores de precipitação permite a elaboração de cenários mais realistas.

A identificação e quantificação da relação entre índices climáticos e a precipitação auxilia na utilização dos mesmos para uma melhor previsão da precipitação dessa região. A existência de uma possível relação entre os índices PDO e AMO e a precipitação local foi observada pelos trabalhos de Silvia e Galvíncio (2011) e Silva (2013), não conseguindo, porém, quantificar as influências individuais de cada índice.

Nesse trabalho foram aplicadas as metodologias do changepoint, Transformada de Ondeletas (TO), Transformada de Ondeletas Cruzadas (XTC) e Análise da Coerência das Ondeletas (WTC) a série de precipitação média da bacia hidrográfica e as séries de índices PDO e AMO. A estação de Iguatu mensura as vazões do Rio Jaguaribe que alimenta o reservatório Orós, um dos principais do 
Estado do Ceará, Brasil. Para obtenção da série de precipitação da bacia foram utilizados dados de chuva dos postos pluviométricos próximos, ponderados pelo método dos polígonos de Thiessen.

O objetivo desse trabalho foi analisar a relação da precipitação média e os índices AMO e PDO buscando uma melhor compreensão dos mecanismos naturais forçantes aos períodos de escassez pluviométrica local. Apresenta como caráter inédito o emprego da metodologia changepoint nesse tipo de análise, juntamente com as ferramentas TO, XTC e WTC, metodologias mais consolidadas para essa aplicação. Os autores ressaltam também que não encontraram trabalhos com aplicação das metodologias XTC e WTC no local de estudo.

\section{Materiais e Métodos}

\section{1 Área de estudo}

A bacia hidrográfica a montante da estação de Iguatu (Fig. 1) possui área total de $20.664 \mathrm{~km}^{2}$, correspondendo a grande parte da área de drenagem do reservatório Orós, sendo essa a estação fluviométrica mais próxima ao reservatório.

\subsection{Dados e padronização das séries}

A partir das bases de dados de postos pluviométricos da Fundação Cearense de Meteorologia e Recursos Hídricos (FUNCEME) e da Superintendência de Desenvolvimento do Nordeste (SUDENE) foi obtida uma série de precipitação diária (jan-1911 a dez-2016) através da ponderação pelo método dos polígonos de Thiessen, considerando estações dentro da bacia hidrográfica e localizadas no entorno com uma distância máxima que não exceda o limite de 1,2 vezes o comprimento longitudinal e horizontal da bacia em questão. A série diária foi agregada mensalmente. $\mathrm{O}$ traçado do Thiessen diário foi realizado através de rotina programada em linguagem $R$.

A aplicação da TO de ondeletas em séries de precipitação é comumente acompanhada de uma normalização da mesma, através do índice de anomalia de precipitação mensal, nesse trabalho foi empregado o índice SPI (Standartized Precipitation Index) na escala temporal de 12 meses, de janeiro a dezembro. A escolha dessa escala se deve a concentração das precipitações da

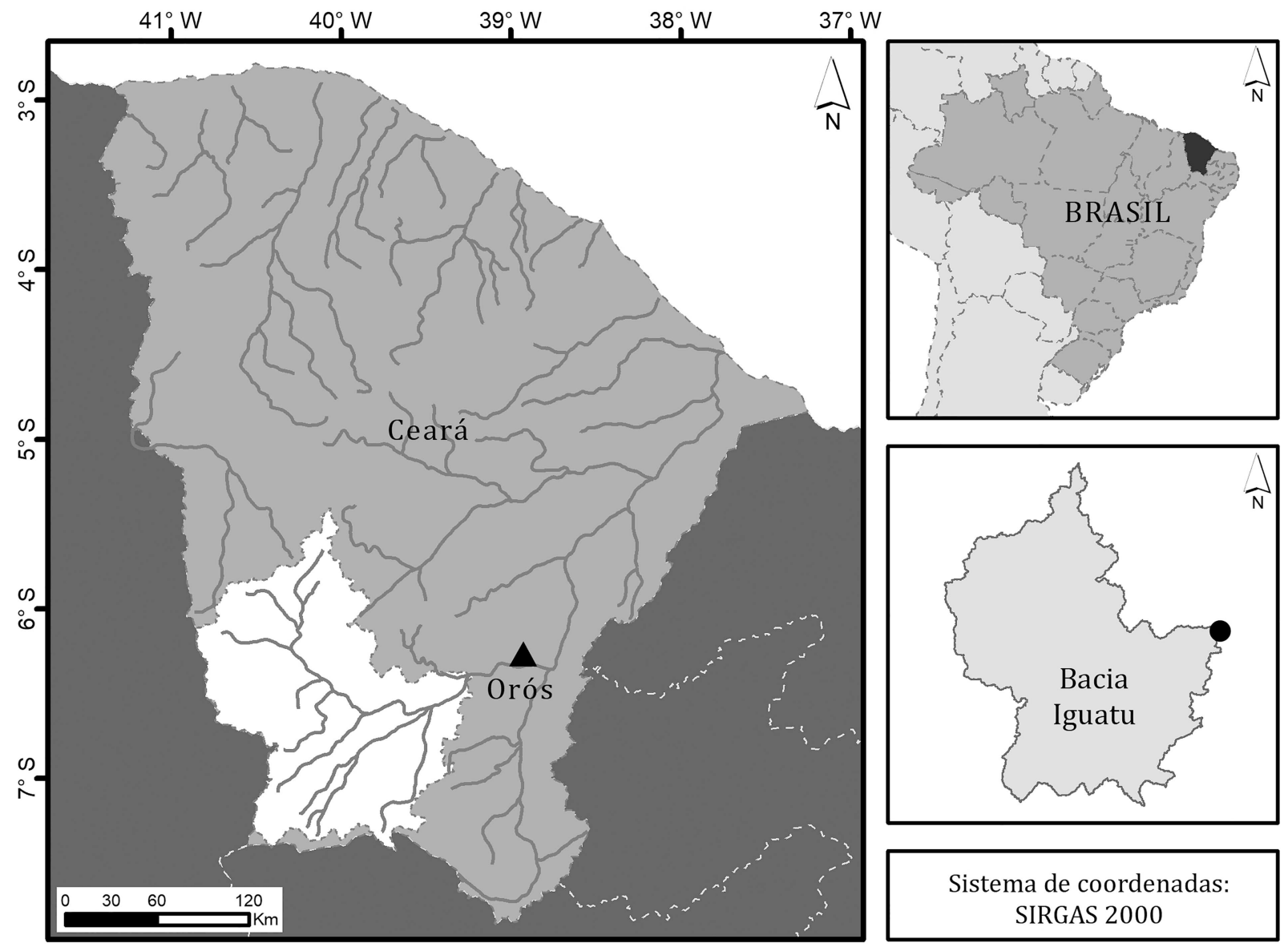

Figura 1 - Localização da bacia hidrológica. 
região de dezembro a maio, com valores médios de precipitação baixos no restante do ano, a utilização de uma escala mensal resultaria em altos valores de SPI para baixos valores de pluviometria ocorridos em meses onde eventos de chuvas são atípicos.

Esse índice é obtido através da aproximação dos valores de precipitação na escala temporal desejada a uma distribuição Gama, obtendo o seu valor através do cálculo da normal padrão inversa relacionado ao valor de probabilidade de não excedência do evento:

$$
\begin{gathered}
H(x)=q+(1-q) G(x) \\
S P I=\phi^{-1}[H(x)]
\end{gathered}
$$

em que $G(x)$ é a distribuição gama incompleta, utilizada para o cálculo da probabilidade acumulada do evento desejado, q é a probabilidade de precipitação igual a zero e $\phi$ a distribuição normal padrão. $\mathrm{O}$ valor de q é usualmente calculado como o número de eventos de precipitação iguais a zero dividido pelo total de observações da escala temporal adotada. Mais informações sobre esse índice consultar Mckee et al. (1993).

O índice AMO foi obtido da base de dados da NOAA (National Oceanic and Atmospheric Administration), na sua versão sem suavização de 1911-2016. Analogamente foram obtidos os dados do índice PDO (A partir dos índices foram obtidas as suas médias anuais, janeiro a dezembro, para padronização da escala temporal com a série de SPI 12.

\subsection{Metodologia}

A TO decompõe uma série temporal $X$, em uma série de funções denominadas ondeletas filhas $\psi(t, s)$ resultantes da dilatação $(s)$ e translação $(t)$ de uma ondeleta mãe (Sivakumar, 2017). É definida em termos de uma integral de convolução entre a série temporal analisada e uma função ondeleta conhecida:

$$
W(t, s)=\frac{1}{\sqrt{s}} \int \psi_{b}\left(\frac{t^{\prime}-t}{s}\right) X(t) d t
$$

O termo $\psi_{b}$ é o conjugado complexo de $\psi$ definido no tempo e escala, $W(t, s)$ é o coeficiente de ondeleta gerado e $\sqrt{ } s$ corresponde a um fator de normalização da energia de cada ondeleta de forma a manter a mesma energia da ondeleta mãe.

A XTC é utilizada para calcular as potências coincidentes entre duas séries temporais, através da equação a seguir (Tang et al. 2014):

$$
W_{n}^{X Y}(t, s)=\frac{1}{s} W_{n}^{X}(t, s) W_{n}^{Y} *(t, s)
$$

em que $W_{n}^{X Y}$ é a transformada cruzada, $W_{n}^{X}$ e $W_{n}^{Y}$ são, respectivamente, a TO da série $X(t)$ e $Y(t)$. A potência da ondeleta cruzada é dado pelo módulo de $W_{n}^{X Y}$.

A WTC é utilizada para identificar as bandas de frequências e intervalos de tempo nos quais as duas séries estão relacionadas (Tang et al. 2014):

$$
R_{n}^{2}(s)=\frac{\left|\left\langle W_{n}^{X Y}(S)\right\rangle\right|^{2}}{\left\langle W_{n}^{X}(s) \vee^{2}\right\rangle\left\langle W_{n}^{Y}(s) \vee^{2}\right\rangle}
$$

em que Rn é a coerência e $<>$ indicam suavização no tempo e escala.

A sincronização entre as fases de algum componente periódico de $X(t)$ em relação ao componente correspondente de $Y(t)$ pode ser representado pela diferença das fases convertido em ângulos no intervalo $[-\pi, \pi]$ e apresentada no espectro de potência através da utilização de setas, conforme a Fig. 2 (Rosch e Schmidbauer, 2014). Para mais informações sobre as metodologias TO, XTC e WTC, consultar as referências mencionadas e os trabalhos de Torrence e Compo (1998) e Torrence e Webster (1999).

Uma das abordagens mais utilizadas para a detecção de pontos de mudanças (changepoints) é através da minimização do custo total, formada pelo somatório do custo associado a adequação da propriedade estatística que se deseja analisar, ao seu conjunto de observações (segmentos):

$$
C_{t}=\sum_{i=1}^{m+1}\left[C\left(y_{\left(\tau_{i-1}\right): \tau_{i}}\right)\right]+\beta f(m)
$$

em que $\mathrm{m}$ é o total de pontos de mudança; $\mathrm{C}(\mathrm{y}(\tau \mathrm{i}-1): \tau i)$ é a função de custo de cada segmento; $\beta \mathrm{f}(\mathrm{m})$ é um fator de penalidade e Ct é o custo total (Haynes; Eckley e Fearnhead, 2014).

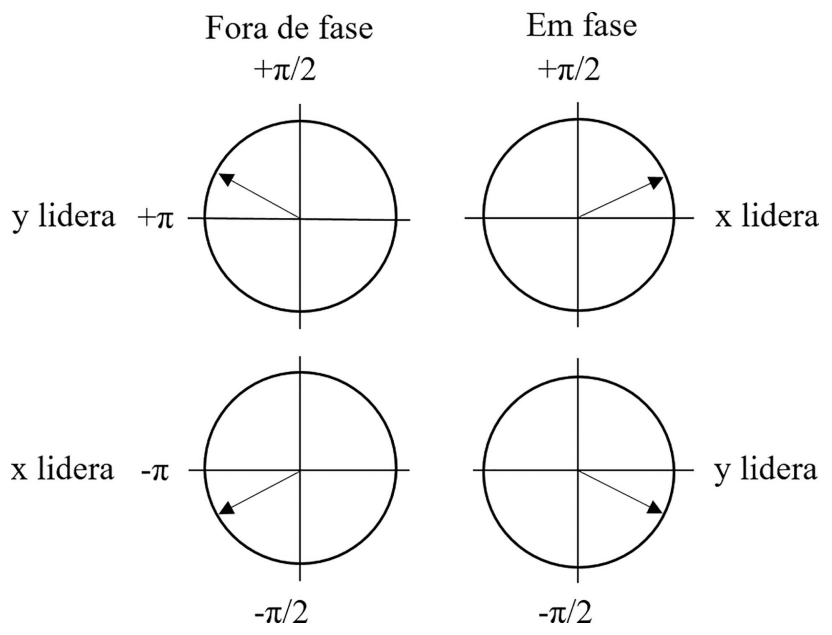

Figura 2 - Diferenças de fases e sua interpretação. Fonte: Adaptado de Rosch e Schmidbauer (2014). 
Essa metodologia de definição do custo depende das premissas impostas sobre a distribuição das observações e também da propriedade estatística da qual se deseja detectar mudanças, visto que o custo do segmento é relacionado a verossimilhança entre a propriedade analisada $e$ as observações contidas.

A adição de um ponto de mudança resulta em uma redução no custo total, dessa forma o fator de penalidade é necessário para evitar a tendência intrínseca ao sobreajuste. A abordagem CROPS (Changepoints for a Range of PenaltieS) permite empregar um intervalo de valores de penalidade ao invés de um valor único, e a análise das segmentações resultantes de diferentes valores de penalidade (Haynes; Eckley e Fearnhead, 2014).

Devido à deteç̧ão de múltiplos changepoints ser uma tarefa computacionalmente intensivas, a literatura apresenta diversas formas de minimização do custo total, entre elas o método exato PELT (Pruned Exact Linear Time) que consegue reduzir o custo computacional removendo das iterações pontos sem alterar os resultados finais (Killick et al. 2012).

A aplicação das metodologias mencionadas foi realizada utilizando as bibliotecas em R WaveletComp (Rosch e Schmidbauer, 2014), para análise por TO, XTC e WTC, e changepoint (Killick e Eckley, 2014), para análise do comportamento das médias, das séries de SPI 12, PDO e AMO mencionadas na seção anterior.

\section{Resultados e Discussão}

Analisando a potência média das Ondeletas do SPI 12 (Fig. 3) é possível observar uma influência de bandas

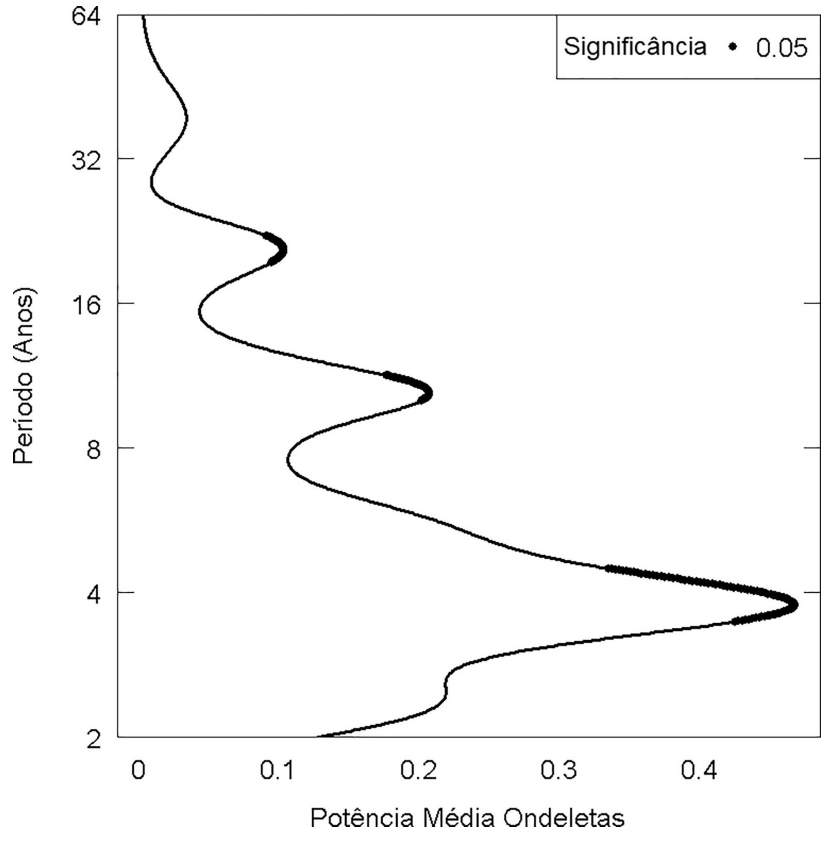

Figura 3 - Potência média das Ondeletas - SPI 12. de frequências entre $\sim 3$ a 4 anos, 9 a 12 anos e 20 a 21 anos, com nível de significância de 0,05. Através do espectro de potência (Fig. 4) nota-se que os períodos que satisfazem um teste a significância de também 0,05 , contornados em preto, não se distribuem igualmente ao longo da série temporal, e que a influência mais relevante de menor frequência está contida no cone de influência e pode ser derivada de efeitos de borda.

A banda de frequência entre 3 a 6 anos apresentou os maiores níveis de potência em dois intervalos de tempo, entre 1911 a 1923, intervalo que apresentou valores mais altos, e entre 1980 a 1985 , e um menor valor ao longo do restante da série. A banda de frequência de 9 a 12 anos apresentou uma influência somente entre 1970 a 1990.

Analisando a potência média das Ondeletas da AMO e o espectro de potência (Figs. 5 e 6a) é possível observar uma influência estatisticamente significante de bandas de frequência entre 20 a 32 anos ao longo de toda a série analisada. Os maiores níveis de potência se localizaram próximo as bandas 3, 4 e 6 anos. Nota-se a influência significativa de uma banda de frequência entre 4 a 10 anos ao longo de toda a série, inicialmente com maiores valores de potência das bandas em torno de 4 anos e ao longo da série valores significativos para bandas de crescente períodos.

Analisando os resultados para a PDO (Figs. 6b e 7) observa-se uma influência ao longo da série toda de uma banda entre 16 a 32 anos. Entre 1911 a 1960 nota-se uma influência de uma banda de 2 a 8 anos, e uma alta potência para banda de 4 a 6 anos entre os anos de 1935 a 1960. Entre 1985 a 2016, a potência da banda de 2 a 8 anos é menor e é possível perceber um maior valor para uma banda entre 8 a 10 anos.

A XTC para o SPI (X) e AMO (Y) (Fig. 8) indica que eles dividem áreas de potência comum nos períodos de 16 a 32 anos ao longo da série toda, iniciando fora de fase e terminando em fase negativa, de 4 a 8 anos entre 1911 a 1960 fora de fase negativa, com altos valores de potência para uma banda de $\sim 4$ anos para os primeiros anos da série, e de 8 a 12 anos (1965 a 2000), alternando entre fora de fase e fase positiva. Esses resultados indicam que a precipitação da área de estudo se relaciona ao índice AMO, principalmente na variabilidade entre 4 a 12 anos e de uma menor frequência ( 20 a 30 anos).

A XTC para o SPI (X) e PDO (Y) (Fig. 9) indica que eles dividem áreas de potência comum nos períodos de 16 a 48 anos entre 1911 a 1980, de 4 a 8 anos entre 1911 a 1960 fora de fase positiva, com altos valores de potência para uma banda de $\sim 4$ anos para os primeiros anos da série e para uma banda $\sim 6$ anos próximo a 1940 , e de 8 a 12 anos (1980 a 2000), em fase negativa. Esses resultados indicam que a precipitação da área de estudo se relaciona ao índice PDO, principalmente na variabilidade entre 4 a 12 anos e de uma menor frequência ( 20 a 30 anos). Notase então uma influência simultânea dos dois índices em intervalos de tempo e períodos similares. 


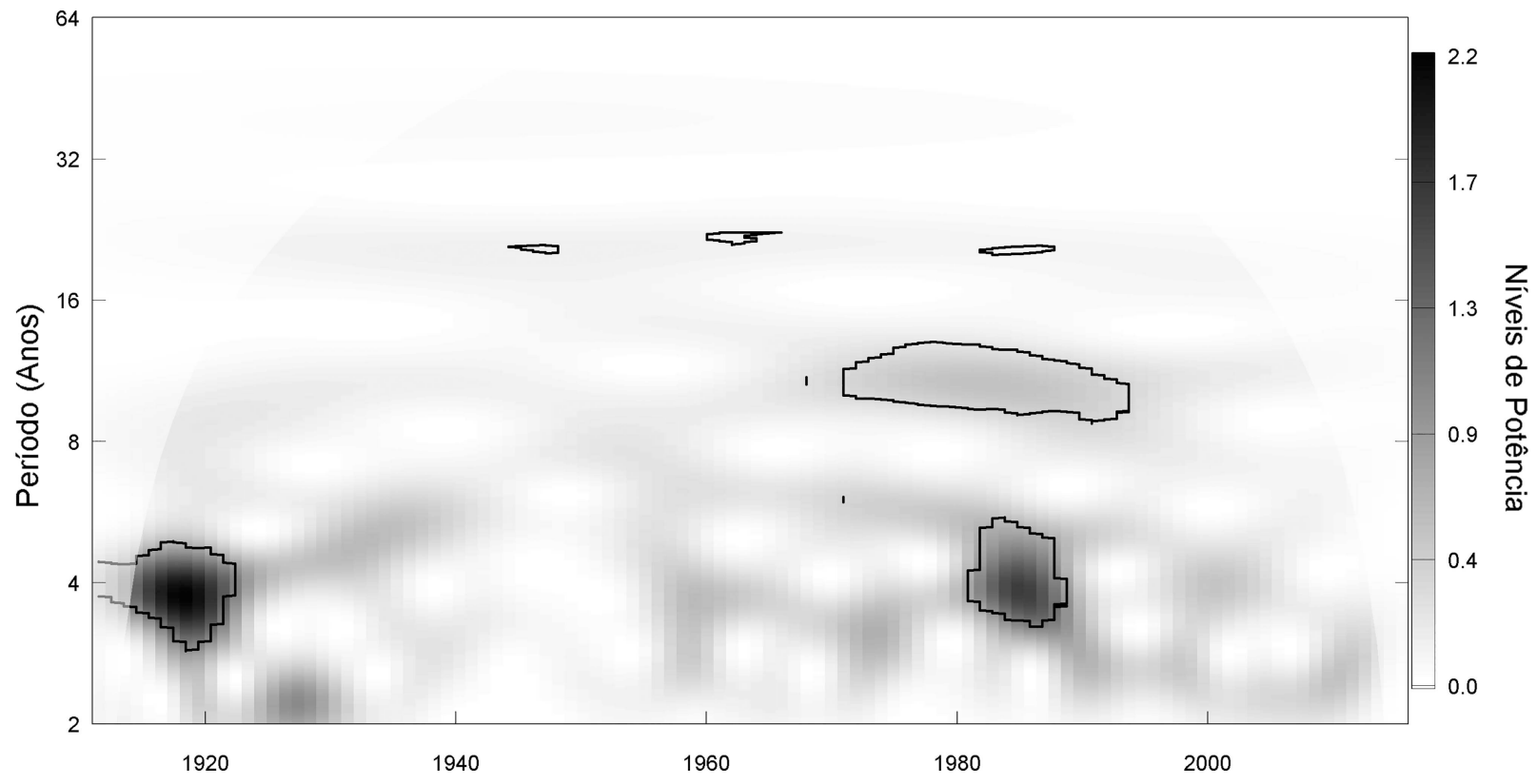

Figura 4 - Espectro de potência SPI 12.

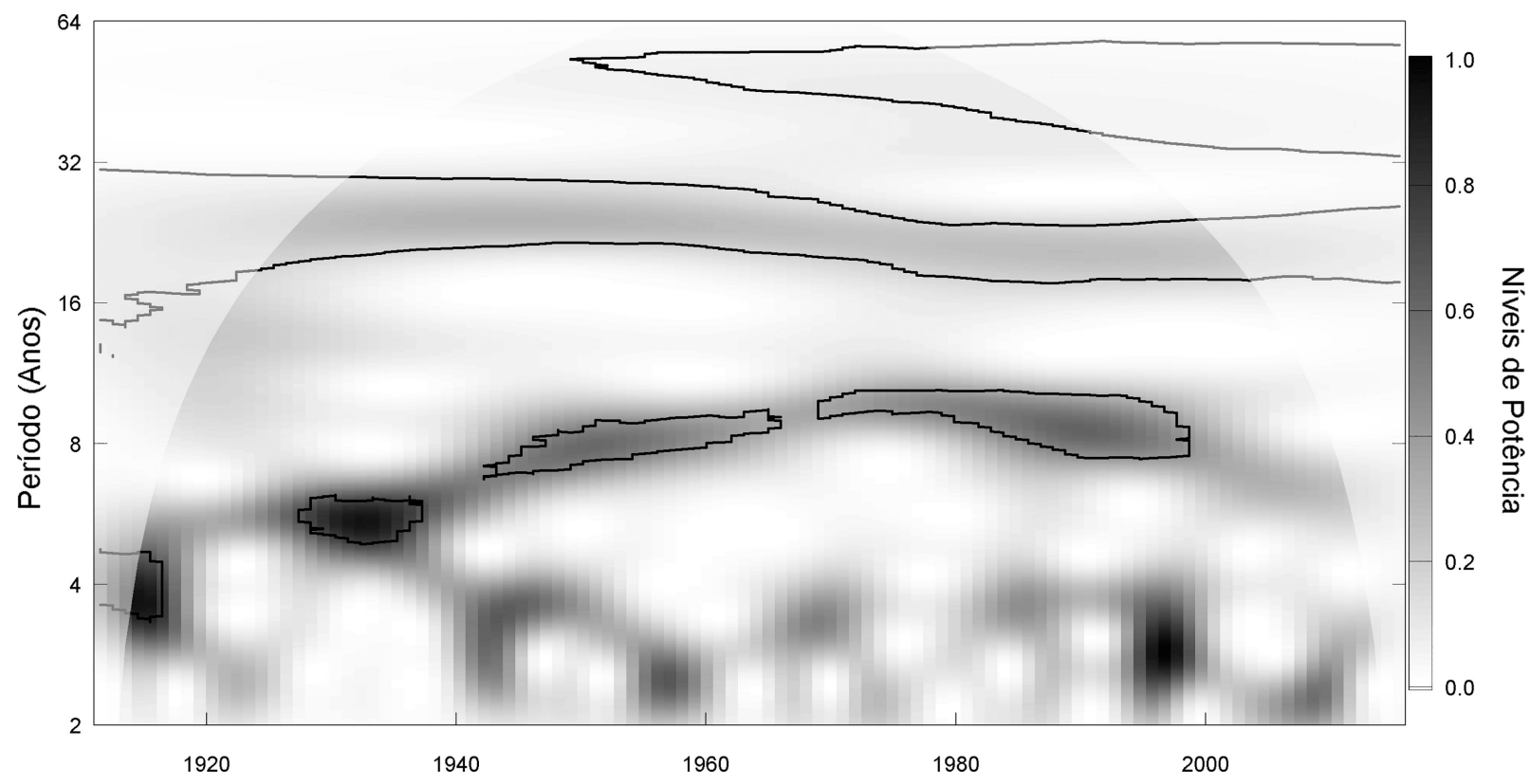

Figura 5 - Espectro de potência AMO.

A WTC entre o SPI e AMO (Fig. 10) indica áreas significativas com altos valores $(>0,8)$ ao longo de quase toda a série, entre 1930 a 1960 para a banda de 4 a 12 anos, de 1975 a 2010 para a bandas entre 16 a 32 e de 10 a 12 anos e para a banda $\sim$ a 4 anos entre 1980 a 1990 . Notase então que a AMO é uma forçante climática que influenciou nas precipitações da área de estudo ao longo de quase todo o período analisado.
A WTC entre o SPI e PDO (Fig. 11) indica áreas significativas, e com valores acima de 0,8 , entre 1911 a 1940 e entre 1950 a 1960 para a banda de 4 a 8 anos, e entre 1945 a 1975 para a banda entre 16 a 24 anos. Nota-se menores valores a partir de 1975 para as bandas mencionadas, com valores altos para as bandas de alta frequência, 2 a 3 anos. Esses resultados indicam então que a PDO teve uma influência na precipitação principalmente entre 1911 a 1975. 

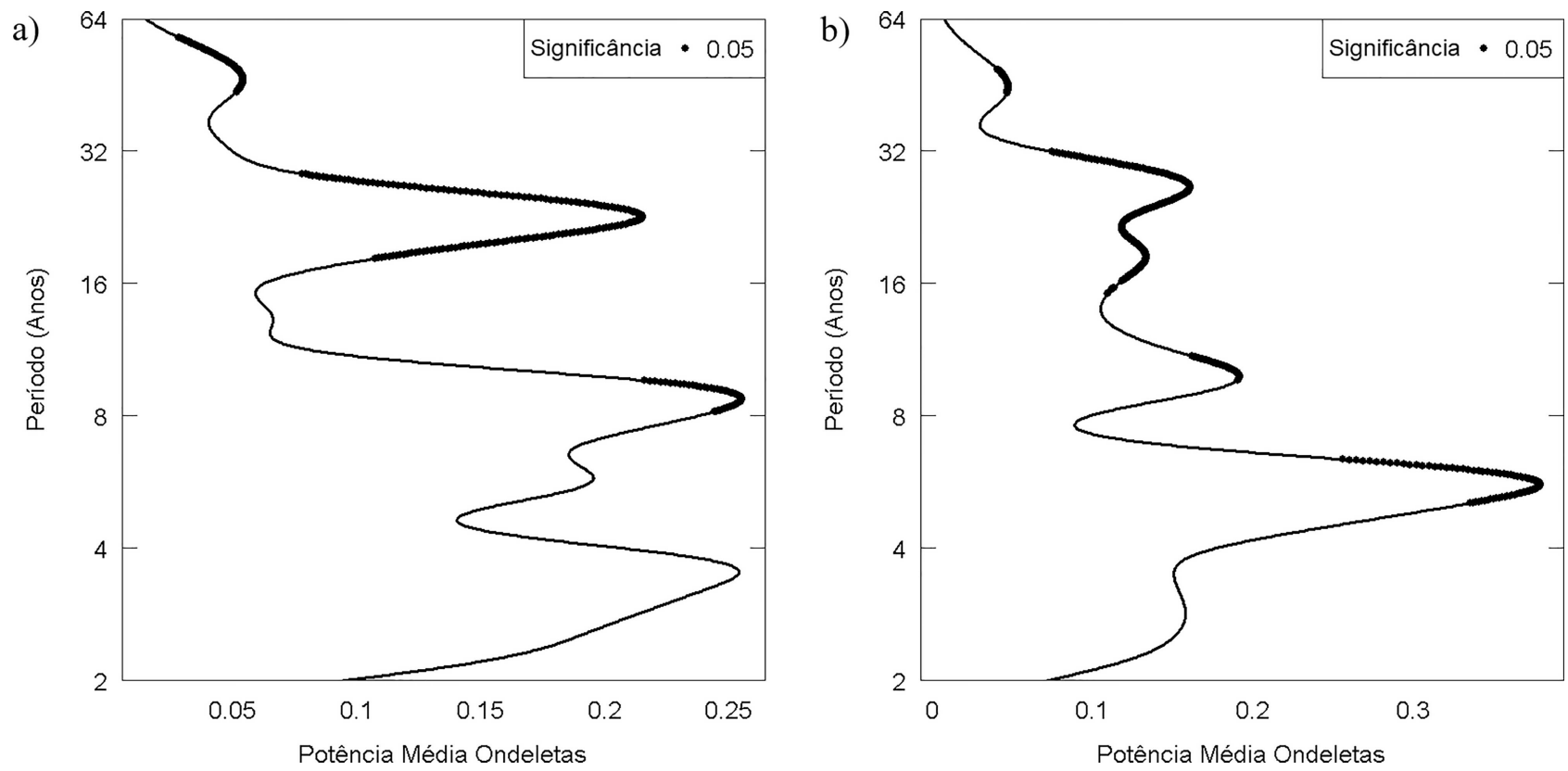

Figura 6 - Potência média das Ondeletas (a) AMO (b) PDO.

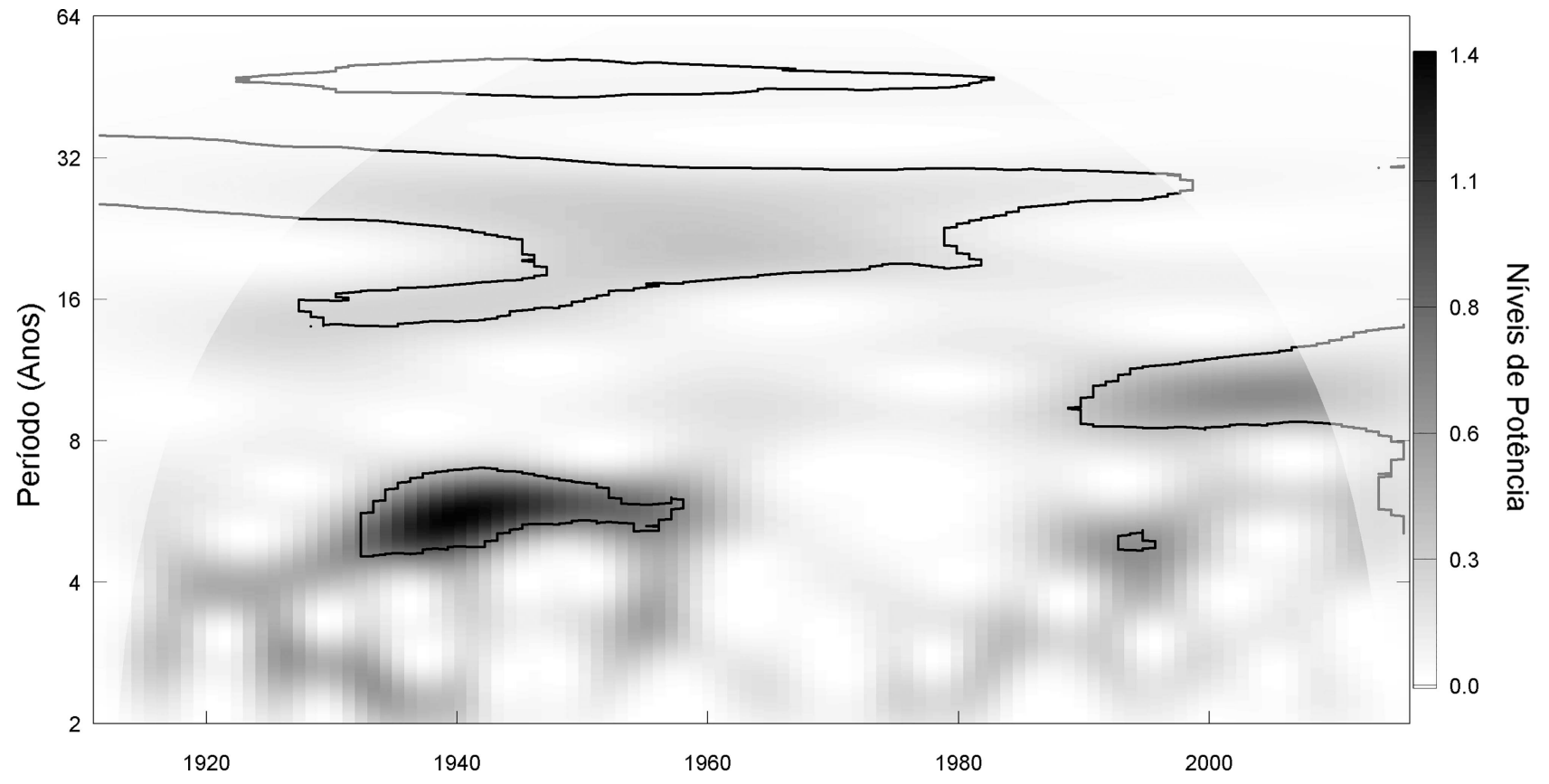

Figura 7 - Espectro de potência PDO.

Devido a correlação das bandas mencionadas com o SPI, um modelo baseado nos dois índices pode ter uma capacidade de previsão do comportamento das precipitações, embora a existência de bandas em fase e fora de fase deve ser analisada para uma correta elaboração do mesmo.

A partir dos resultados obtidos pela metodologia do changepoint (Fig. 12), podemos perceber uma maior variação da $\mathrm{PDO}$ em comparação com a AMO, a AMO apresenta uma segmentação em apenas 6 períodos, com uma maior variação entre os anos de 1960 a 1995. Para a PDO foram detectados um total de 17 pontos de mudança, onde entre 1930 a 1950 nota-se uma maior amplitude de variação.

Comparando os valores das segmentações obtidas da AMO e PDO com o SPI, nota-se, entre 1911 a 1915, um valor médio de SPI e PDO próximo a zero $(-0,13$ e - 0,17$)$ e da AMO próximo a -0,2. Entre 1916 a 1926, um SPI 


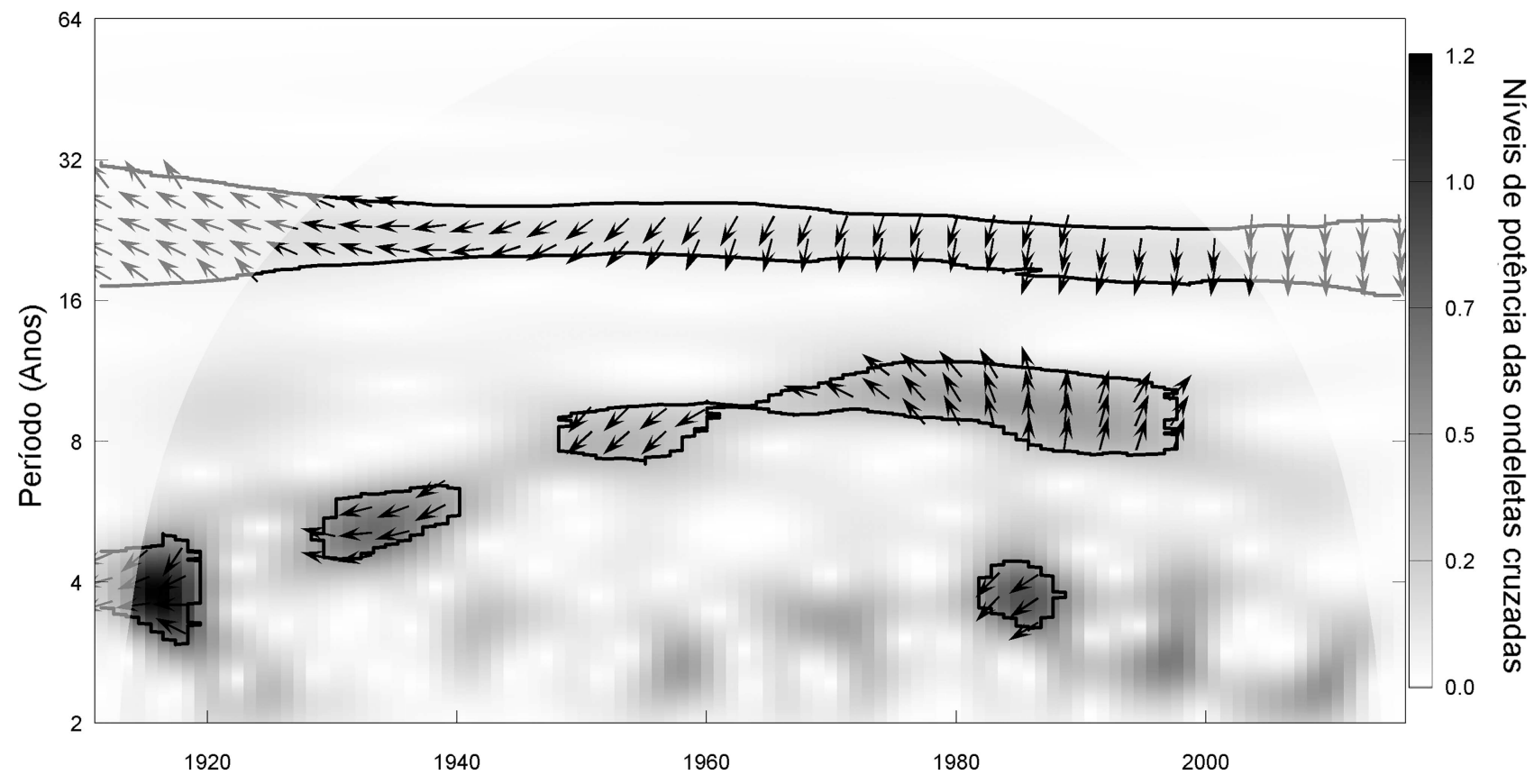

Figura 8 - Espectro de potência XTC - SPI e AMO.

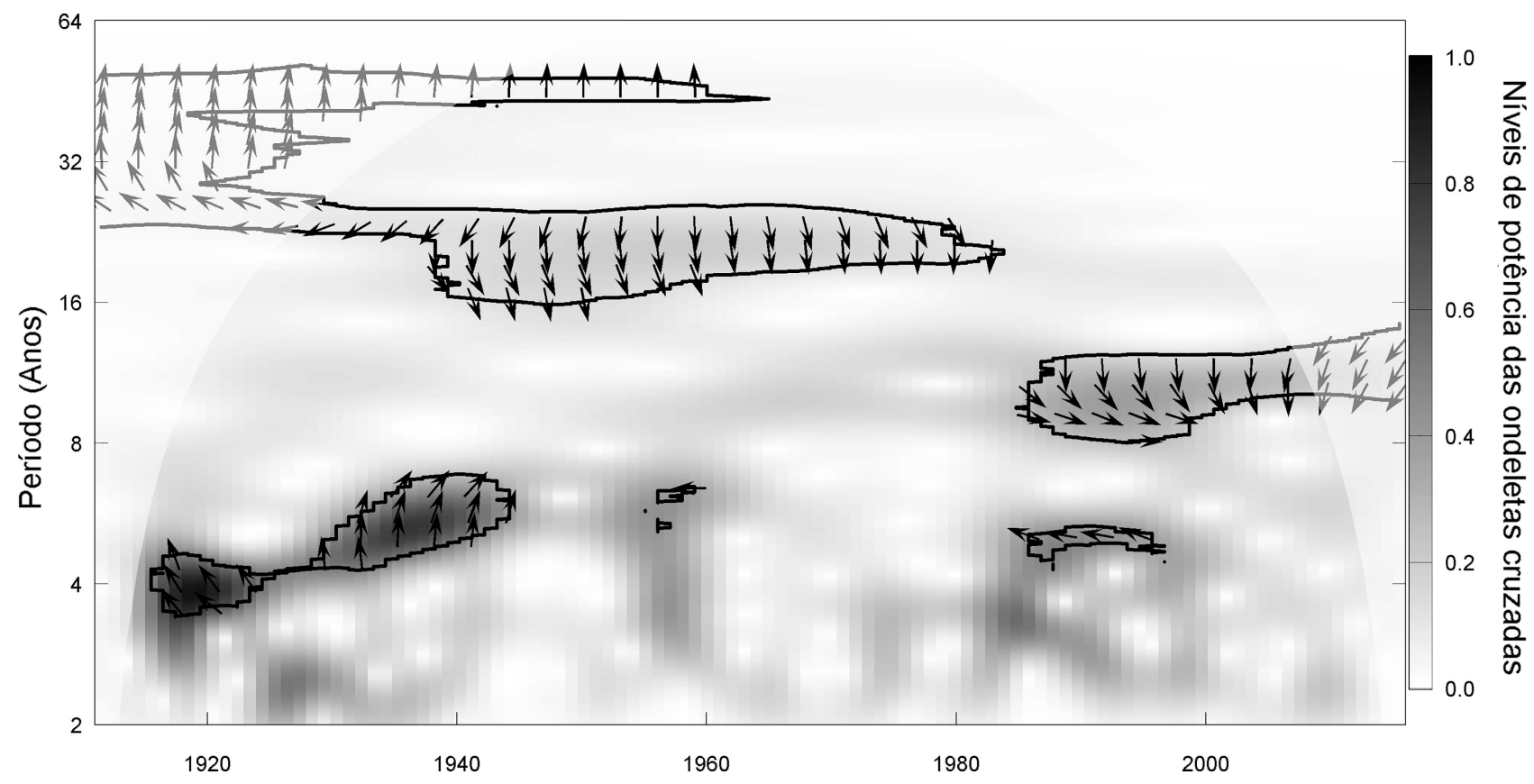

Figura 9 - Espectro de potência XTC - SPI e PDO.

médio próximo a 1, exceto para o ano de 1919, e PDO e AMO em fase negativa (-1 e -0,2). Entre 1927 a 1935, SPI próximo a zero $(0,21), \mathrm{AMO}$ em fase positiva com valor médio igual a 0,11 e PDO alternando entre valores positivos e negativos $(0,5,-1,13$ e 0,90$)$, com valores positivos durante a maior parte do tempo. Entre 1936 a 1957, SPI médio igual a $-0,52$, AMO na mesma fase positiva e uma alta variação dos valores de médios de PDO (de -1,4 a
2,07), com uma duração maior dos valores negativos. Os anos 1958 e 1959 apresentaram valores baixos de SPI $(-1,74)$ e PDO e AMO em fase positiva $(0,11$ e 0,4$)$. De 1960 ao final da série, nota-se uma transição da AMO da fase positiva para negativa $(0,11,-0,09,-0,34$ e $-0,15)$, atingindo o menor valor médio da série analisada, e retornando a um valor positivo a partir de $1995(0,15)$, valores médios negativos para a PDO entre 1961 a $1976(-0,68)$, 


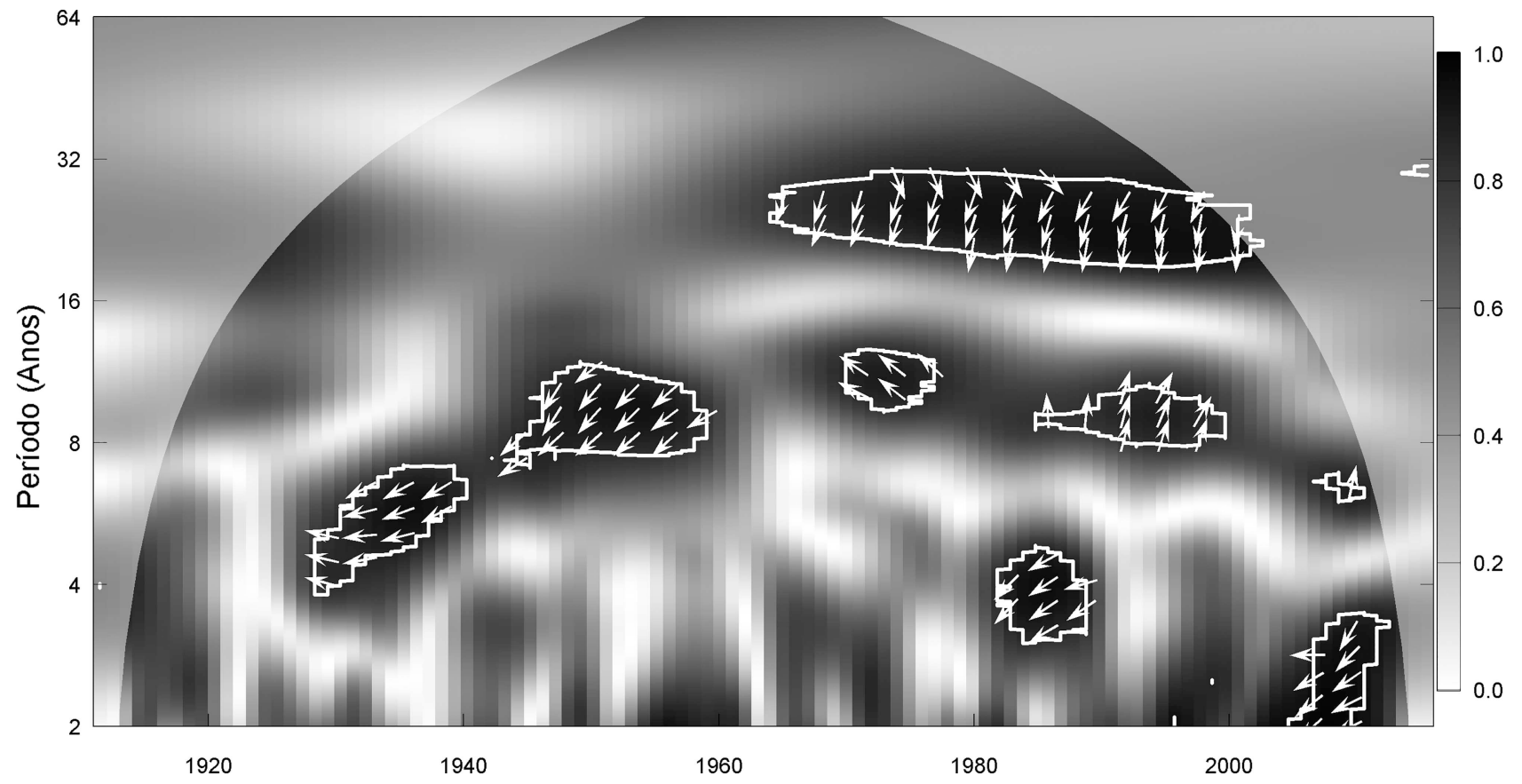

Figura 10 - Espectro de potência WTC - SPI e AMO.

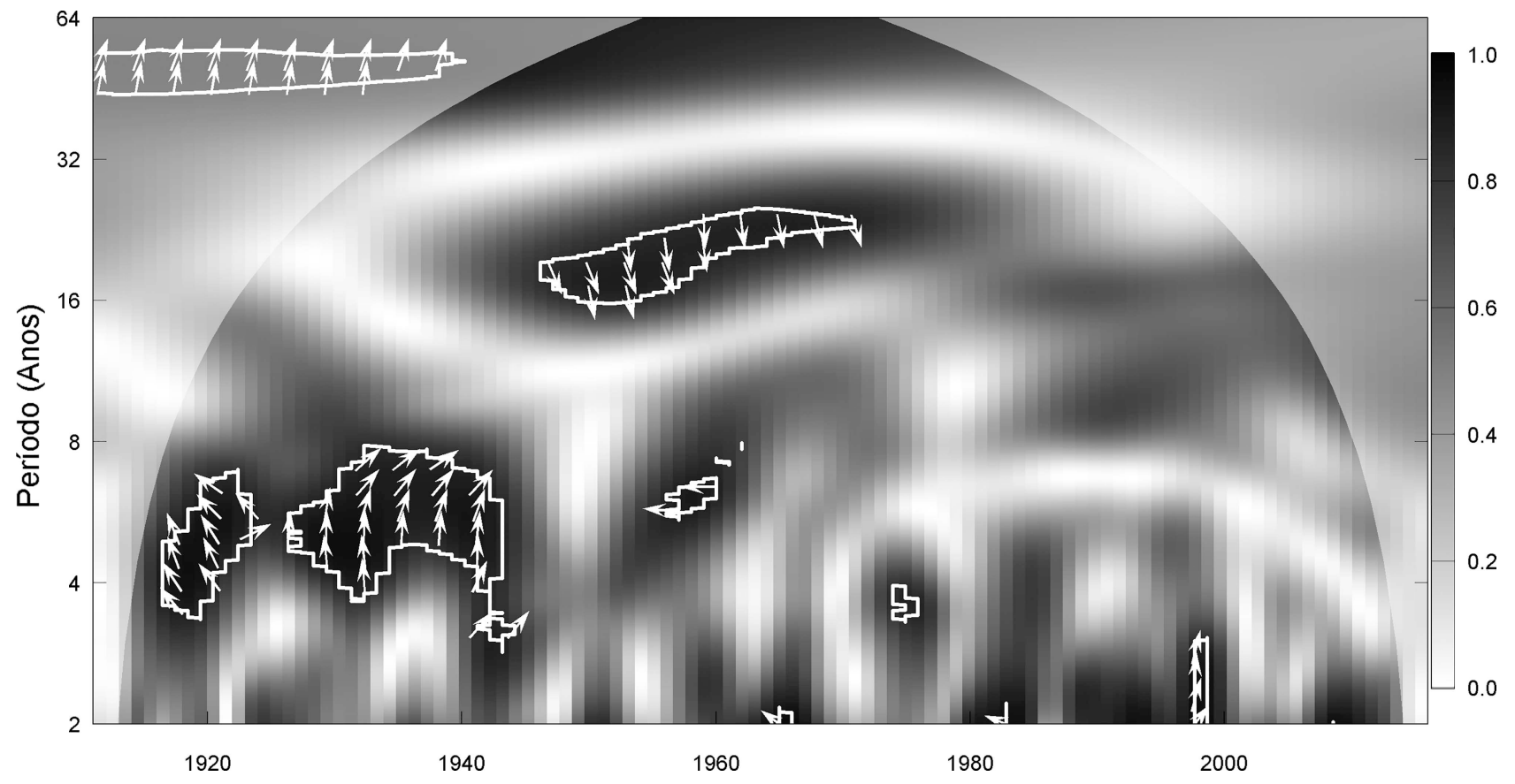

Figura 11 - Espectro de potência WTC - SPI e PDO.

1989 a $1991(-0,78)$ e 1998 a $2013(-0,65$ e -1,46), positivos de 1977-1988, 1992-1997 e 2014-2016 (0,39, 0,55 e $0,63)$,e um SPI próximo a zero $(0,21,-0,08$ e 0,16$)$, durante a maior parte do tempo exceto para o pico do ano de 1973 (2,35), a depressão de 1983 (-2,03), um período positivo entre 1984 a $1989(1,17)$ e os períodos negativos de $1990-1993$ e $2012-2016(-0,68$ e $-1,07)$.
É possível observar valores positivos (negativos) de SPI nos períodos que o AMO e PDO estão conjuntamente negativos (positivos), indicando uma correlação negativa entre o SPI e as duas variáveis, esse comportamento pode ser visualizado mais explicitamente nos períodos entre 1915 a 1925, 1958 a 1959 e 2012 a 2016. 

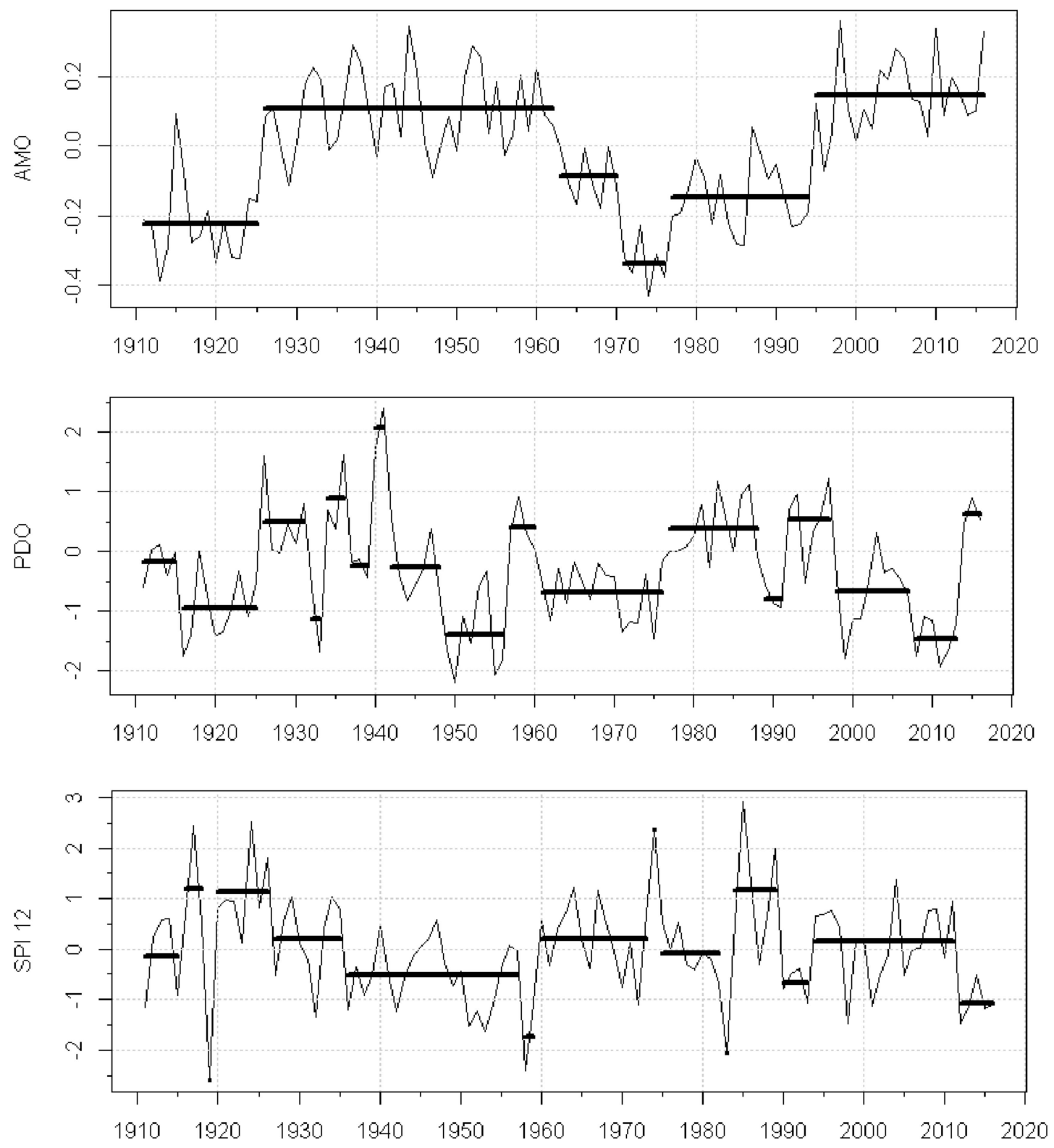

Figura 12 - Resultados changepoint AMO, PDO e SPI.

A maior variação da PDO comparativamente a AMO e a diferença de fases observada anteriormente entre os componentes periódicos dificulta a visualização, e quantificação, da influência dessas oscilações na precipitação da região do estudo e indica também um possível lag entre o comportamento da AMO e a sua influência na precipitação.

\section{Conclusões}

Empregando as metodologias explicitadas foi possível detectar a influência conjunta das variações de TSM do Oceano Atlântico e Pacífico nas precipitações médias da região estudada e concluir que períodos com fases simultaneamente positivas e negativas da PDO e da AMO temos comportamento das precipitações mais definidos, espera-se maiores valores de precipitação para períodos cujo a PDO e AMO estão conjuntamente negativos e menores valores para a situação oposta.

A aplicação das metodologias permitiu também detectar correlação entre as bandas de 4 a 8 anos e de 16 a 32 anos dos índices PDO e AMO e o comportamento do SPI indicando que um modelo baseado nos dois índices pode ter uma capacidade de previsão do comportamento das precipitações, embora deva levar em consideração a existência de bandas em fase e fora de fase para uma correta elaboração do mesmo. 
A existência de bandas fora de fase identificadas pela metodologia WTC entre o SPI e a AMO indica um possível lag no relacionamento dessas variáveis.

\section{Agradecimentos}

O primeiro autor agradece a Coordenação de Aperfeiçoamento de Pessoal de Nível Superior (CAPES) e a Agência Nacional das Águas (ANA) pelo suporte financeiro através da bolsa de mestrado relacionada ao edital ANA Mudanças Climáticas e Recursos Hídricos.

\section{Referências}

CASTRO, B.C.A.; SOUZA FILHO, F.A.; SILVEIRA, C.S. Análise de Tendências e Padrões de Variação das Séries Históricas de Vazões do Operador Nacional do Sistema (ONS). Revista Brasileira de Recursos Hídricos, v. 18, n. 4, p. 19-34, 2013.

ENFIELD, D.B.; MESTAS-NUÑEZ, A.M.; TRIMBLE, P.J. The Atlantic multidecadal oscillation and its relation to rainfall and river flows in the continental US. Geophysical Research Letters, v. 28, n. 10, p. 2077-2080, 2001.

HAYNES, K.; ECKLEY, I.A.; FEARNHEAD, P. Efficient penalty search for multiple changepoint problems. arXiv preprint arXiv:1412.3617, 2014.

FUNCEME, Fundação Cearense de Meteorologia e Recursos Hídricos. Volume Armazenado. FUNCEME, Fortaleza, 2017.

KILLICK, R; ECKLEY, I.A. Changepoint: An R Package for Changepoint Analysis. Journal Of Statistical Software, v. 58, n. 3, p.1-19, 2014.

KILLICK, R.; FEARNHEAD, P.; ECKLEY, I.A. Optimal Detection of Changepoints With a Linear Computational Cost. Journal Of The American Statistical Association, v. 107, n. 500, p.1590-1598, 2012.

ROSCH, A.; SCHMIDBAUER, H. WaveletComp: A guided tour through the R-package. p. 1-38, 2014.
TORRENCE, C.; COMPO, G.P. A Practical Guide to Wavelet Analysis. Bulletin of the American Meteorological Society, v. 79, n. 1, p. 61-78, 1998.

TORRENCE, C.; WEBSTER, P.J. Interdecadal changes in the ENSO-monsoon system. Journal of Climate, v. 12, n. 8 PART 2, p. 2679-2690, 1999.

SILVA, D.F.; GALVÍNCIO, J.D. Estudo da Influência da Oscilação Decadal do Pacífico no Nordeste do Brasil. Revista Brasileira de Geografia Física, v. 4, n. 4, p. 665-676, 2011.

SILVA, D.F. Influência da variabilidade interdecadal do clima associada ao ENOS sobre o Estado do Ceará. Revista IberoAmericana de Ciências Ambientais, v.4, n.2, p.8698, 2013.

SILVEIRA, C.S.; SOUZA FILHO, F.A.; AQUINO, S.H.S.; SILVA, S.M.O. (2015). Variabilidade Climática e Planejamento em Recursos Hídricos. In: Uso da informação climática em múltiplas escalas temporais para o planejamento do setor hidroelétrico brasileiro. Org. por Souza Filho, Francisco de Assis de; Silveira, Cleiton da Silva. Fortaleza: Expressão Gráfica e Editora, p. 13-26.

MANTUA, N.J.; Hare, S.R., ZHANG, Y.; WALLACE, J.M.; FRANCIS, R. C. A. Pacific interdecadal climate oscillation with impacts on salmon production. Bulletin of the American Meteorological Society, v. 78, n. 6, p. 1069-1080, 1997.

MCKEE, T.B.; DOESKEN, N.J.; KLEIST, J. The relationship of drought frequency and duration to time scales. Proceedings of the 8th Conference on Applied Climatology, v. 17, n. 22, p. 179-183, 1993. Boston, MA: American Meteorological Society.

TANG, C.; CHEN, D.; CROSBY, B. T.; PIECHOTA, T.C.; WHEATON, J.M. Is the PDO or AMO the climate driver of soil moisture in the Salmon River Basin, Idaho? Global and Planetary Change, v. 120, p. 16-23, 2014.

This is an Open Access article distributed under the terms of the Creative Commons Attribution License, which permits unrestricted use, distribution, and reproduction in any medium, provided the original work is properly cited. Legendas: 